\title{
Optimizing Prices and Periods in Time-of-use Electricity Tariff Design Using Bilevel Programming
}

\author{
Maria João Alves ${ }^{1}$, Carlos Henggeler Antunes ${ }^{2}$, and Inês Soares ${ }^{3}$ \\ 1 CeBER and Faculty of Economics, University of Coimbra / INESC Coimbra, \\ Portugal mjalves@fe.uc.pt \\ 2 INESC Coimbra, Dept. of Electrical Engineering and Computers, University of \\ Coimbra, Portugal ch@deec.uc.pt \\ 3 INESC Coimbra, Portugal inesgsoares@gmail.com
}

\begin{abstract}
In this paper, a comparison is made between two bilevel programming models to design time-of-use tariffs in the electricity retail market. The upper-level objective function consists of the maximization of the retailer's profit and the lower-level problem relates to the minimization of the consumer's cost. In the first model, the periods in which prices apply are pre-defined and the aim is to determine the price values. In the second model, which is developed for the first time in this paper, both the periods and prices are decision variables, thus leading to a very large search space for the upper-level problem due to the number of combinations periods-prices. For the model with variable periods, a hybrid approach combining a genetic algorithm for the upper-level search with a mixed-integer linear programming solver to obtain optimal solutions to the lower-level problem is herein developed. Computational results comparing the two models are presented.
\end{abstract}

Keywords: Bilevel optimization · Genetic algorithm · Mixed-integer linear programming model · Time-of-use pricing · Electricity retail market . Demand response

\section{Introduction}

Major changes are underway in the electricity sector, namely regarding the evolution to smart grids, the increasing share of renewable sources, the dissemination of electric vehicles, the deployment of distributed storage and the empowerment of consumers/prosumers. Retail electricity markets are very competitive and retail companies should design appropriate pricing schemes to offer to consumers, who are increasingly sensitive to the need to manage consumption patterns in an optimal manner by considering cost and comfort dimensions in their energy decisions. Energy service companies and grid operators provide automated home energy management systems (HEMS), which manage consumption on the

\footnotetext{
^ corresponding author
} 
consumer's behalf according to his preferences (e.g., time slots for appliance operation). In general, retailers buy energy in spot markets (e.g., day ahead) or through bilateral contracts. These prices seen by the retailer are increasingly influenced by the grid status and the generation mix required to satisfy demand. So, time-of-use prices have been increasingly adopted, thus fostering a "load follows supply" paradigm in such a way that benefits can be obtained for all players in the energy supply chain (generators, grid operators, retailers and consumers).

There is a hierarchical interplay between a retailer and consumers. The retailer establishes time-of-use prices (which can be valid for a long-term contract, e.g. one year, or be dynamic, e.g. announced one day ahead) to maximize profits. The consumer reacts by managing his consumption to minimize the electricity bill, which can be facilitated by the use of HEMS. Therefore, the design of timeof-use pricing schemes, i.e. specifying variable energy prices and the periods in which they apply, considering the demand response, is of utmost importance for the electricity retail business. This problem has been dealt with bilevel optimization (BLO) models, which are well suited to represent this hierarchical decision setting. The retailer is the leader, who decides first by setting prices for given periods, and the consumer is the follower, who reacts to these prices by determining the appliance schedule that optimizes his cost function. Although the retailer is the first to play, he must consider the consumer's reaction because it affects the retailer's profit.

In BLO, the (lower-level) follower's optimization problem is nested in the constraints of the (upper-level) leader's problem. BLO models are, in general, very difficult to handle theoretically, methodologically and computationally [1]. Most approaches reported in the literature for designing time-of-use tariffs are devoted to computing the energy prices for pre-defined periods (e.g., along one day for daily cycle prices). This problem has been addressed by several authors, e.g. in [2],[3], [4], [5], [6] and [7] among others, the last one using a trilevel model.

The problem of designing time-of-use pricing schemes becomes more realistic if, in addition to the price values, the periods in which prices apply are also determined as a result of the optimization. However, this problem becomes much more complex to solve. A general framework of models to optimize prices and periods can be set as follows - models M1 to M4 - being the price values decision variables in all of them; a certain discretization of the planning horizon is considered (e.g., 24 hours discretized in 96 units of 15 minutes each) and the periods to define the prices are ordered sets of time units (t.u.):

M1) periods are pre-defined;

M2) periods are variable, each one consisting of a set of contiguous t.u. (i.e., each period is defined by a start t.u. and an end t.u.), with a pre-specified number of different periods (which implies a maximum number of different prices for the whole planning horizon);

M3) a price is assigned to each t.u., but imposing a pre-specified maximum number of different prices for the whole planning horizon;

M4) no restrictions, i.e. each t.u. may have a different price. 
Model M4 is the most general one (prices and periods are totally free) and model M1 is the most restricted one (periods are pre-defined). Model M3 is more general than model M2: in M3, prices may change from each t.u. to the next, while in M2 prices may change only by the pre-defined number of periods. In other words, M3 is an extension of M2, looking at each period as a set of possibly non-contiguous t.u..

These four time-of-use pricing models may have further variations due to additional constraints on periods (e.g., minimum number of $t$. $u$. in each period) or prices (e.g., minimum difference between different prices). Further constraints may be introduced in the time dimension or in price magnitudes.

Models M1 and M2 are more realistic to be implemented in electricity retail markets, namely having in mind consumer's acceptance. By increasing the number of combinations of prices and periods, model M2 offers the retailer the expectation of increasing profits with respect to the ones obtained with model M1 (which is a particular case of M2). This paper is devoted to model M2: a maximum number of different periods is specified; the problem consists of determining the start and the end $t . u$. for each period and the corresponding price value. A comparison with model M1 is carried out.

Our previous works have been devoted to model M1 [4]-[6] and M3+M4 [8] using hybrid approaches combining a meta-heuristic (particle swarm optimization or genetic algorithm) to perform the upper-level search for prices and a solver to obtain the solution to the lower-level mixed-integer linear programming (MILP) problem for each price setting. Although models M3 and M4 are interesting from a conceptual perspective, in practice they may induce an excessive change of prices that probably would not be accepted by consumers, or even regulatory authorities, as a viable tariff option.

It should be noticed that the problem of designing time-of-use electricity tariffs falls into the broad category of price setting problems ([9],[10]) which includes, e.g., the toll setting problem (the problem of defining highway tolls, where costumers want to minimize their individual generalized travel costs). In these problems, the leader typically seeks to maximize revenues (or profits) raised from taxes or tariffs, while consumers specify consumption or production levels aiming to minimize costs. Therefore, the present study may also act as a lever for future works in pricing setting problems aiming to balance demand along time, which have not yet been addressed in the literature using optimization models. An example may be the definition of happy hours and drink/food prices in bars and restaurants.

In section 2, the main concepts of BLO are presented and bilevel models for price setting problems in the electricity retail market are outlined, considering i) variable prices only (M1 model) and ii) variable prices and periods of time (variable period model - M2 model). In section 3, a genetic algorithm for the variable period model is described. Numerical results comparing the two models are presented in Section 4 and the main conclusions are drawn in section 5. 


\section{$2 \quad$ Bilevel modelling of electricity prices}

A general BLO problem can be formulated as follows:

$$
\begin{array}{rl}
\max _{x \in X} & F(x, y) \\
\text { s.t. } & G(x, y) \leq 0 \\
& y \in \underset{y \in Y}{\arg \min }\{f(x, y): g(x, y) \leq 0\}
\end{array}
$$

where $x \in \mathbb{R}^{n_{1}}$ is the vector of variables controlled by the leader - the decision maker at the upper-level problem - and $y \in \mathbb{R}^{n_{2}}$ is the vector of variables controlled by the follower - the decision maker at the lower-level problem.

In a bilevel problem, the decision process is sequential as the leader makes his decisions first by setting the values of the variables $x$. Then, the follower reacts by choosing the $y$ values that optimize his objective function on the feasible solutions restricted by the fixed $x$. The bilevel problem is the leader's problem. However, the leader must incorporate into the optimization process the reaction of the follower because this affects the leader's objective value and even the feasibility of the solution. It is difficult to find global optimal solutions to bilevel optimization problems due to their inherent non-convexity. Even the linear bilevel problem is NP-hard [1].

In Alves et al. [4], a bilevel problem was considered to model the interaction between the electricity retailer (leader) and a cluster of consumers (follower) with similar consumption and demand response profiles. The retailer buys energy in the wholesale market and wants to determine the prices $x_{i}$ to be charged to the consumers in $I$ pre-defined periods $P_{i}(i=1, \ldots, I)$ of a planning horizon discretized into $T$ time units $(t=1, \ldots, T)$, in order to maximize his profit. The consumer aims to minimize the electricity bill, by reacting to the electricity prices communicated by the retailer and deciding on the operation of controllable appliances. In [4] only shiftable appliances were considered, in addition to a base load not deemed for control (e.g., tv set, oven, fridge, etc.). Shiftable appliances are typically cyclic loads, such as dishwashers or laundry machines, whose operation cycle can be shifted in time but not interrupted once initiated.

In Soares et al. [6], the bilevel model in [4] was extended by including other types of controllable appliances with different physical features and type of control: in addition to shiftable appliances, a thermostatic load (air conditioning system) and interruptible appliances have been modelled in the lower-level problem. Interruptible appliances are loads whose operation can be interrupted provided that the necessary amount of energy is supplied during a required time slot (e.g. charge of an electric vehicle). In both studies ([4], [6]) the lower-level optimization model is a mixed-integer programming problem, which can be solved by an exact MILP solver (for instance, CPLEX) for each instantiation of the upper-level variables $x$.

In the current study, we consider bilevel models with a consumer's problem including $J$ shiftable appliances $(j=1 \ldots J)$ and $K$ interruptible appliances $(k=$ 
$1 \ldots K)$ as the controllable loads. The consumer wants to determine the times of the operation of these loads in order to minimize the electricity bill, ensuring that the operation of each load is within a specified comfort time slot defined by a start t.u. and an end $t . u$. within the planning horizon $\{1, \ldots, T\}:\left[t_{j}^{1}, t_{j}^{2}\right], j=1 \ldots J$ and $\left[t_{k}^{1}, t_{k}^{2}\right], k=1 \ldots K$, respectively, for shiftable and interruptible appliances. Each shiftable appliance $j$ has a load diagram specifying the power $\left(q_{j r}^{\text {shif }}\right)$ required at each stage $r$ (one t.u.) of its operation cycle with duration $d_{j}$. Each interruptible appliance $k$ has the requirement that the energy $E_{k}$ should be supplied during the given comfort time slot, being $q_{k}^{\text {int }}$ the power requested at each t.u. when the load is operating. The electricity bill includes an energy component (cost of the energy consumed by all loads) and a power component (the retailer defines multiple levels of contracted power, $P_{l}^{\text {cont }}, l=1 \ldots L$, with different prices $e_{l}$ and the consumer pays for the power level corresponding to the peak).

The electricity prices in each period $P_{i}$ are controlled at the upper-level: $x_{i}$, $i=1, \ldots, I$, where $I$ is the number of periods $P_{i}$.

The lower-level decision variables are binary variables: $v_{k t}$, which specify, for each interruptible appliance $k$, whether it is operating or not at each time unit $t$ of the respective comfort time slot; in the case of shiftable appliances, the binary variables are $w_{j r t}$, which further include the index $r$ to specify the stage of the operation cycle in which the load is operating at each $t$. These binary variables define auxiliary real variables $p_{t}, \forall t$, which represent the power requested from the grid by all loads: shiftable, interruptible and also a (constant) base load $b_{t}$ not deemed for control. These variables, together with the electricity prices set by the leader, define the cost of energy for the consumer: $\sum_{i=1}^{I} \sum_{t \in P_{i}} x_{i} p_{t}$. Binary decision variables $u_{l} \in\{0,1\}, l=1 \ldots L$, are also used to model the power component, identifying the peak power level the consumer should be charged for in the whole planning horizon: $\sum_{l=1}^{L} e_{l} u_{l}$ (the constraints ensure that only one $u_{l}$ is equal to 1 ).

The formulation of the lower-level combinatorial optimization problem is:

$$
\begin{aligned}
& \min _{p, u} f=\sum_{i=1}^{I} \sum_{t \in P_{i}} x_{i} p_{t}+\sum_{l=1}^{L} e_{l} u_{l} \\
& \text { s.t. } \\
& \quad p_{t}=b_{t}+\sum_{j=1}^{J} \sum_{r=1}^{d_{j}} q_{j r}^{\text {shif }} w_{j r t}+\sum_{k=1}^{K} q_{k}^{\text {int }} v_{k t}, \quad t=1, \ldots, T \\
& \quad \sum_{r=1}^{d_{j}} w_{j r t} \leq 1, \quad j=1, \ldots, J ; t=t_{j}^{1}, \ldots, t_{j}^{2} \\
& w_{j(r+1)(t+1)} \geq w_{j r t}, \quad j=1, \ldots, J ; r=1, \ldots, d_{j}-1 ; t=t_{j}^{1}, \ldots, t_{j}^{2}-1 \\
& \sum_{t=t_{j}^{1}}^{t_{j}^{2}} w_{j r t}=1, \quad j=1, \ldots, J ; r=1, \ldots, d_{j}
\end{aligned}
$$




$$
\begin{aligned}
& \sum_{t=t_{j}^{1}}^{t_{j}^{2}-d_{j}+1} w_{j 1 t} \geq 1, \quad j=1, \ldots, J \\
& w_{j r t}=0, \quad j=1, \ldots, J ; r=1, \ldots, d_{j} ; t<t_{j}^{1} \vee t>t_{j}^{2} \\
& \sum_{t=t_{k}^{1}}^{t_{k}^{2}} q_{k}^{\text {int }} v_{k t}=E_{k} \quad k=1, \ldots, K \\
& v_{k t}=0, \quad k=1, \ldots, K ; t<t_{k}^{1} \vee t>t_{k}^{2} \\
& \sum_{l=1}^{L} u_{l}=1 \\
& p_{t} \leq \sum_{l=1}^{L} P_{l}^{\text {cont }} u_{l}, \quad t=1, \ldots, T \\
& u_{l} \in\{0,1\}, \quad l=1, \ldots, L \\
& w_{j r t} \in\{0,1\}, \quad j=1, \ldots, J ; r=1, \ldots, d_{j} ; t=t_{j}^{1}, \ldots, t_{j}^{2} \\
& v_{k t} \in\{0,1\}, \quad k=1, \ldots, K ; t=t_{k}^{1}, \ldots, t_{k}^{2}
\end{aligned}
$$

where constraints (2) define the power requested at each $t$ by all loads, constraints (3) - (7) model the operation of the shiftable appliances, (8) - (9) model the operation of the interruptible appliances and (10) - (11) model the contracted power.

For a given $x=\left(x_{1}, \ldots, x_{I}\right)$, the lower-level problem is a MILP problem with a large number of binary variables and constraints. The genetic algorithm used to perform the upper-level search calls the MILP solver CPLEX to solve the lower-level problem (as a black-box).

\subsection{Bilevel model with pre-defined periods (M1)}

The bilevel model M1, in which only the prices $x_{i}$ are decision variables for the retailer because the periods $P_{i}$ are pre-specified (models in [4]-[6]), can be stated as follows:

$$
\begin{gathered}
\max _{x} F=\sum_{i=1}^{I} \sum_{t \in P_{i}} x_{i} p_{t}+\sum_{l=1}^{L} e_{l} u_{l}-\sum_{t=1}^{T} \pi_{t} p_{t} \\
\text { s.t. } \\
\quad \underline{x} \leq x_{i} \leq \bar{x}, \quad i=1, \cdots, I \\
\frac{1}{T} \sum_{i=1}^{I} \bar{P}_{i} x_{i} \leq x^{A V G}
\end{gathered}
$$

$$
(1)-(14)
$$

where $\pi_{t}$ is the energy price seen by the retailer in the spot market at each $t \in\{1, \ldots, T\}$ and $\bar{P}_{i}$ denotes the amplitude of $P_{i}$, i.e. $\bar{P}_{i}=P_{i}^{2}-P_{i}^{1}+1$ 
where $P_{i}^{1}, P_{i}^{2}$ delimit each period $P_{i}, i \in\{1, \ldots, I\}$ (the start and end t.u., respectively). In order to enforce market competitiveness of retailer prices, the upper-level constraints impose minimum and maximum values on prices in each period $P_{i}$ and an average price $\left(x^{A V G}\right)$ value during the whole planning horizon. In the present study, we consider the same minimum and maximum price values throughout the planning horizon ( $\underline{x}$ and $\bar{x}$, respectively).

\subsection{Bilevel model with variable periods (M2)}

In model M1, the prices to be established in each pre-defined period were the only decision variables. Model M2 builds on model M1 to offer the retailer the possibility of optimizing not just the price values but also the periods in which they apply. This is accomplished by defining a number of periods (which, for instance, may result from regulatory obligations) and determining their optimal start and end t.u. within the planning horizon. The imposition of a number of periods $I$ constrains the maximum number of different price values.

Each period $P_{i}$ is defined by a start t.u. $\left(P_{i}^{1}\right)$ and an end t.u. $\left(P_{i}^{2}\right)$. Thus, the upper-level variables of $\mathrm{M} 2$ are: $x_{i}, P_{i}^{1}$ and $P_{i}^{2}, i=1, \ldots, I$. Two sets of constraints characterize the upper-level problem of Model M2: constraints on prices $x$ (the same as in M1 but with $P_{i}^{1}$ and $P_{i}^{2}$ being decision variables) and constraints that ensure continuity of the periods. Only either $P_{i}^{1}$ or $P_{i}^{2}$ need to be considered, because these variables depend on each other $\left(P_{i+1}^{1}=P_{i}^{2}+\right.$ 1 ), but both are represented in the model below to improve clarity. Continuity constraints ensure that the first period starts at $t=1$, the last one ends at $t=T$, and the periods are chained: $P_{1}^{1} \leq P_{1}^{2}=P_{2}^{1}-1, \ldots, P_{I-1}^{2}+1=P_{I}^{1} \leq P_{I}^{2}=T$. For instance, suppose that one t.u. is $15 \mathrm{~min}$ and the planning horizon starts at 00:00 $\mathrm{h} ; P_{1}^{1}=1$, meaning that period $P_{1}$ includes, at least, the first $15 \mathrm{~min}$ of the day; if, for instance, $P_{1}^{2}=4$, then the first period is [00:00, 01:00[ $\mathrm{h}$ and the second period starts at $P_{2}^{1}=5$, which means that $P_{2}$ includes at least $t=5$, i.e., [01:00, 01:15[ h. The model M2 can be stated as follows:

$$
\begin{aligned}
\max _{x, P^{1}, P^{2}} F & =\sum_{i=1}^{I} \sum_{t \in\left[P_{i}^{1}, P_{i}^{2}\right]} x_{i} p_{t}+\sum_{l=1}^{L} e_{l} u_{l}-\sum_{t=1}^{T} \pi_{t} p_{t} \\
\text { s.t. } & \underline{x} \leq x_{i} \leq \bar{x}, \quad i=1, \cdots, I \\
& \frac{1}{T} \sum_{i=1}^{I}\left(P_{i}^{2}-P_{i}^{1}+1\right) x_{i} \leq x^{A V G} \\
& P_{1}^{1}=1 ; \quad P_{I}^{2}=T \\
& P_{i+1}^{1}=P_{i}^{2}+1, \quad i=1, \cdots, I-1 \\
& P_{i}^{2} \geq P_{i}^{1}, \quad i=1, \cdots, I \\
& P_{i}^{1}, P_{i}^{2} \quad \text { integer }, \quad i=1, \cdots, I
\end{aligned}
$$




$$
\begin{aligned}
& \min _{p, u} f=\sum_{i=1}^{I} \sum_{t \in\left[P_{i}^{1}, P_{i}^{2}\right]} x_{i} p_{t}+\sum_{l=1}^{L} e_{l} u_{l} \\
& \text { s.t. } \quad(2)-(14)
\end{aligned}
$$

Other conditions may be imposed on the periods, which we call aggregation constraints, e.g. the following ones.

- The length of each period must be a multiple of a given number of units of time. Consider, for instance, that the planning horizon is discretized in units of quarter-hour (which is generally used for measurements in power systems and enables a fine grain analysis of appliance operation) and the prices must be defined for periods that are multiple of half-hour or one hour (e.g., for regulatory reasons). Therefore, the amplitude of each period $\left(P_{i}^{2}-P_{i}^{1}+1\right)$ must be multiple of 2 or 4 , respectively. For a multiple of $\beta$ units of time, and provided that $T$ is multiple of $\beta$, the following constraints are included:

$$
\begin{gathered}
P_{i}^{2}-P_{i}^{1}+1=\beta k_{i}, \quad i=1, \cdots, I \\
k_{i} \text { integer, } 1 \leq k_{i} \leq \frac{T}{\beta}, \quad i=1, \cdots, I
\end{gathered}
$$

- Each period must have a minimum length $C$, where $C$ is a constant:

$$
P_{i}^{2}-P_{i}^{1}+1 \geq C, \quad i=1, \cdots, I
$$

In the present study, we have considered constraints (15) with $\beta=2$, which also ensure a minimum length of $C=2$. Since the quarter-hour is the t.u. considered, the periods are then multiple of half-hour.

The electricity prices charged to the consumer $\left(x_{i}, i=1, \ldots, I\right)$ and the prices seen by the retailer in the spot market $\left.\left(\pi_{t}, t=1, \ldots, T\right)\right)$ are presented in $€ / \mathrm{KWh}$. So, an adequate scale factor $\alpha$ is applied in the upper and lower-level objective functions of $\mathrm{M} 1$ and $\mathrm{M} 2$ to convert the prices into the t.u. used in these models. That is, $x_{i}$ and $\pi_{t}$ are replaced by $\alpha x_{i}$ and $\alpha \pi_{t}$, respectively. In this study, $\alpha=1 / 4$.

\section{A genetic algorithm for the variable period model}

We aim at comparing results obtained for models M1 and M2. The algorithm presented in [4] has been adapted to deal with model M1 considering prices with 4 decimal places (as it is usual in electricity bills presented to consumers) instead of real numbers. The algorithm consists of a genetic algorithm (GA) to deal with the upper-level search combined with CPLEX to find the optimal solution to the lower-level problem for each $x$ vector. The individuals dealt with by the GA are the price vectors $x=\left(x_{1}, x_{2}, \ldots, x_{I}\right)$. The upper-level constraints of M1 are ensured by a repair routine [4], which has been adjusted in the present work to prices with a fixed number of decimal places and is also used in the approach developed for model M2. This routine is briefly described below. 
The approach developed for the variable period model M2 is also a hybrid GA-solver. The individuals are composed by two vectors, one for prices (as in model M1) and the other for periods. Since the start t.u. of each period is the end t.u. of the previous period $+1\left(P_{i}^{1}=P_{i-1}^{2}+1\right)$, the periods are represented only by their end t.u. : $P^{2}=\left(P_{1}^{2}, P_{2}^{2}, \ldots, P_{I-1}^{2}, T\right)$ with increasing integer values $P_{i}^{2}<P_{i+1}^{2}$. This vector has dimension $I$, but the last component is fixed to $T$. These two vectors define each upper-level solution and are illustrated in Fig. 1.

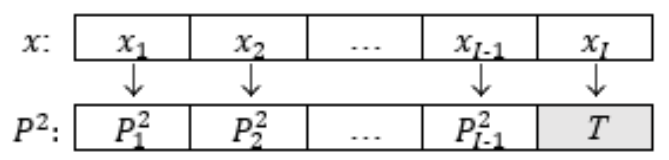

Fig. 1. Encoding of an upper-level solution in model M2

A population of $N$ individuals $\left(x^{n}, P^{2, n}\right), n=1, \ldots, N$ evolves throughout $G$ iterations of the GA. The vector of start t.u. $P^{1, n}$ corresponding to $P^{2, n}$ is: $P^{1, n}=\left(1, P_{1}^{2, n}+1, \ldots, P_{I-1}^{2, n}+1\right)$. For each individual, the lower-level problem of M2 with $P^{1}=P^{1, n}, P^{2}=P^{2, n}$ and $x=x^{n}$ is exactly solved. Let $y^{n}=\left(p^{n}, u^{n}\right)$ be the optimal solution obtained for this lower-level instance (vector $p$ of power required by all load operation and vector $u$ of binary variables that determine the contracted power). Each solution is then evaluated by the upper-level objective function $F\left(x^{n}, P^{1, n}, P^{2, n}, y^{n}\right)$, which gives its fitness.

The general description of the GA is presented below.

Step 1 - Create the initial population with $N$ individuals $(n=1, \ldots, N)$

- Generate each $P^{2, n}: I-1$ different integer numbers are randomly drawn; these values are then sorted by increasing order. In order to satisfy the time limits and the aggregation constraints (15), multiples of $\beta$ are generated in the range $(1, T-1) ; P_{I}^{2, n}=T, \forall n$.

- Generate each $x^{n}$ : a real number with 4 decimal places is randomly generated in $[\underline{x}, \bar{x}]$ for each component $x_{i}^{n} ; x^{n}$ is then repaired to satisfy also the average price constraint (repair routine).

Step 2 - Obtain the lower-level solutions: for each individual, the $P^{1, n}$ vector associated with $P^{2, n}$ is defined and the lower-level problem is solved for $\left(x^{n}, P^{1, n}, P^{2, n}\right)$ to obtain $y^{n}$. Compute its fitness value, $F^{n}$.

While the maximum number of generations $G$ is not achieved do Step 3 - Selection and Reproduction

- Select $N$ pairs of individuals for being parents: one parent is randomly chosen and the other is the winner of a binary tournament with replacement.

- In order to ensure that all $P^{2}$ values will satisfy the aggregation constraints, their scale is changed by dividing all values by $\beta$, which will be recovered at the final of the reproduction phase; after scaling, $P_{I}^{2}=\tilde{T}=T / \beta$. 
- For each pair of parents $\left(P^{2^{\prime}}, x^{\prime}\right)$ and $\left(P^{2^{\prime \prime}}, x^{\prime \prime}\right)$, an one-point crossover operator is separately applied to $P^{2}$ and $x$. For $P^{2}$, the last component (equal to $\tilde{T}$ ) never changes.

- Apply mutation to each offspring with a given probability of changing each gene of $x$ and of $P^{2}$. The mutation of an $x_{i}$ consists of adding or subtracting a positive perturbation randomly generated in the range $[0,0.2(\bar{x}-\underline{x})]$, ensuring that $x_{i}$ remains within bounds. The mutation of $P_{i}^{2}$ consists of adding or subtracting 1 t.u., ensuring that $1 \leq P_{i}^{2}<\tilde{T}$. In this study, the mutation probability is 0.05 as in [4].

- For each offspring $P^{2}$ vector , sort $P_{i}^{2}(i=1, \ldots, I-1)$ by increasing order and adjust it to contain no repeated values, since there are no periods with duration 0 ; convert $P^{2}$ to the original scale (see Fig. 2 for an example).

- Repair each $x$ vector of the offspring to also satisfy the average price constraint and keeping 4 decimal places (repair routine).

- Obtain the lower-level solutions for the offspring as in Step 2.

Step 4 - Replacement

Form the next population by copying the solution with best $F$ obtained thus far (which is either in the current population or in the offspring) and by performing $N-1$ binary tournaments without replacement between individuals of the current population and the offspring population.

\section{End While}

Return the solution with the highest fitness $F$.

Fig. 2 illustrates the reproduction process (without mutation) of two 6-period vectors, $P^{2^{\prime}}$ and $P^{2^{\prime \prime}}$, for a planning horizon of $T=96 t$.u. of quarter-hour and $\beta=2$ (periods should be multiple of half-hours). The vectors $P^{2^{\prime}}$ and $P^{2^{\prime \prime}}$ at the top of Fig. 2 have already been scaled to half-hour units (thus, $\tilde{T}=48$ ). After applying crossover and sorting by ascending order the values of the offspring $P^{2}\left(P_{i}^{2}, i=1, \ldots, I-1\right)$, if there are two equal values then the second one is incremented by 1 . In this example, this only happened once for value 27 . If a modified value becomes equal to the next, then this process is repeated until all duplicate values are eliminated. If the second to the last value $\left(P_{I-1}^{2}\right)$ is equal to the last one $(\tilde{T})$, the chromosome is discarded and another one must be generated.

In this work, a one-point crossover operator has been used both for $P^{2}$ and $x$ vectors. A geometric crossover operator for the prices $x$ had been used in the study [8] for models M3 and M4, but the results with the GA were not encouraging.

The repair routine implemented in both algorithms (for M1 and M2 models) ensures that prices are within bounds $\left(\underline{x} \leq x_{i} \leq \bar{x}, i=1, \ldots, I\right)$ and satisfy the average price constraint $\left(\frac{1}{T} \sum_{i=1}^{I} \bar{P}_{i} x_{i} \leq x^{A V G}\right.$ with $\left.\bar{P}_{i}=P_{i}^{2}-P_{i}^{1}+1\right)$. Since the aim is to maximize the retailer's profit, the repair operations attempt to set prices as close as possible to satisfying the average price constraint as an equality, keeping all values with a fixed number of $d$ decimal places (we have been working with $d=4$ ). The repair routine operates as follows. Firstly, the $x_{i}$ 


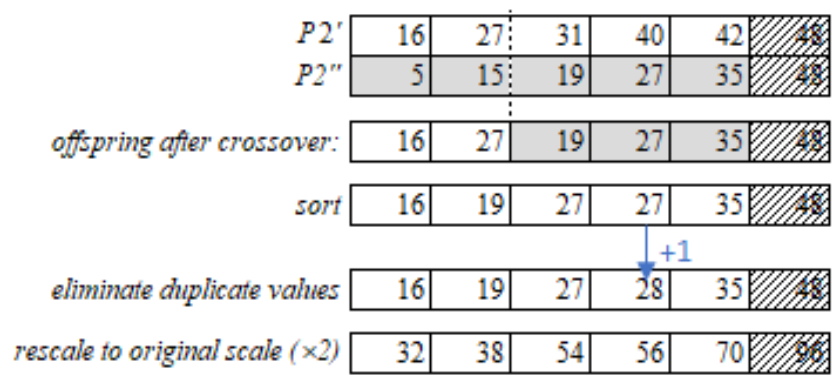

Fig. 2. Crossover and adjustment of periods

are truncated to $d$ decimal places and pushed into bounds. Let $A$ be the set of indices $i$ of $x$ that can still change; initially, $A=\{1, \ldots, I\}$. The following cycle is repeated until a valid $x$ is obtained or $A=\emptyset$ :

(1) $x_{i} \leftarrow \operatorname{trunc}\left(x_{i}+\delta, d\right), \forall i \in A$, with

$$
\delta=\frac{T x^{A V G}-\sum_{i=1}^{I} \bar{P}_{i} x_{i}}{\sum_{i \in A} \bar{P}_{i}}
$$

(2) if all $x_{i}$ are within the bounds, then stop: a valid $x$ has been obtained; otherwise, push into the closest bound $(\underline{x}$ or $\bar{x})$ any $x_{i}$ that is out of bounds, $A \leftarrow A \backslash\{i\}$ and return to (1) if $A \neq \emptyset$.

\section{Results}

A case study has been used to compare results obtained with the M1 and M2 models using the respective hybrid GA algorithms. A 24-hour planning horizon (starting at 00:00 h) is considered, which is discretized into t.u. of quarter-hour, leading to a planning horizon $\{1, \ldots, 96\}$. The consumer's problem includes five controllable appliances: three shiftable loads (dishwasher, laundry machine and clothes dryer) and two interruptible loads (electric water heater and electric vehicle). The data concerning the consumer's problem were obtained from actual audit information and some values were estimated; they can be found in the Supplementary Material of [6], including the operation cycles of the loads, the comfort time slots allowed for the operation of each load, the base load, the contracted power levels and their costs, as well as the prices seen by the retailer at the spot market. These data define a lower-level problem with 559 binary variables.

Six periods of time $P_{i}, i=1, \ldots, 6$, are considered for defining the electricity prices to be charged by the retailer to the consumer (as in [6]). In model M1, the pre-defined periods $\left[P_{i}^{1}, P_{i}^{2}\right], i=1, \ldots, 6$, are: [1-28], [29-44], [45-56], [5772], [73-84], [85-96]. These periods reproduce realistic time-of-use tariff schemes being currently used and induce good solutions to the retailer (thus, imposing more challenges to M2 to yield better solutions). In model M2, the periods 
are decision variables. In both models, the minimum and maximum prices that can be charged to the consumer are: $\underline{x}=0.08 € / \mathrm{kWh}$ and $\bar{x}=0.35 € / \mathrm{kWh}$. The average price over the entire planning horizon cannot exceed $x^{A V G}=0.18$ $€ / \mathrm{kWh}$.

We started by running the algorithms (for M1 and M2) considering different parameterizations of the population size $(N)$ and number of generations $(G)$ in order to choose a configuration that provides a satisfactory compromise between the quality of the solution obtained vs. the computation time needed. The following parameterizations were attempted for $N \times G: 30 \times 100,30 \times 200$, $40 \times 150$, the last two requiring similar computation effort, since both require solving 6000 lower-level MILP problems, which is the most demanding part of the computational effort. Ten independent runs were performed with M1 and M2 for each parameterization. In order to allow a better comparison, an equal rand-seed (seed used for the generation of random numbers) was considered for runs with the same index. For instance, run $k$ started with rand-seed $r_{k}$ for all parameterizations $N \times G$ in M1 and M2. The best, worst, average and standard deviation of the retailer's profit $(F)$ obtained over the 10 runs for each parametrization in each model are reported in Table 1 . All $F$ values are in $€$ and refer to a cluster of 1000 consumers with similar consumption and demand response profiles. The best values for each model are highlighted in bold.

Table 1. Results of different combinations of the population size and number of generations for M1 and M2

\begin{tabular}{lccc|ccc}
\hline & \multicolumn{3}{c}{$\mathrm{M} 1(N \times G)$} & \multicolumn{3}{c}{$\mathrm{M} 2(N \times G)$} \\
$F$ values & $30 \times 100$ & $30 \times 200$ & $40 \times 150$ & $30 \times 100$ & $30 \times 200$ & $40 \times 150$ \\
\hline Maximum & 6038.67 & $\mathbf{6 0 3 9 . 2 4}$ & $\mathbf{6 0 3 9 . 2 7}$ & 6145.12 & $\mathbf{6 1 5 1 . 1 6}$ & 6145.75 \\
Minimum & 6026.96 & $\mathbf{6 0 3 5 . 9 9}$ & 6028.68 & $\mathbf{6 0 7 0 . 7 2}$ & $\mathbf{6 0 7 0 . 7 2}$ & $\mathbf{6 0 7 0 . 7 2}$ \\
Average & 6034.49 & $\mathbf{6 0 3 7 . 5 7}$ & 6036.80 & 6116.18 & $\mathbf{6 1 2 1 . 1 4}$ & 6108.95 \\
Stand.dev. & 3.387 & 0.963 & 2.773 & 26.930 & 27.813 & 21.981 \\
\hline
\end{tabular}

From this experiment, we can observe (Table 1):

- Better retailer's profit can be obtained when the retailer can set prices and periods (model M2) over setting prices only (model M1) (1.85\% improvement in the best cases). This result was expected, because the M2 solution space, say $S(\mathrm{M} 2)$, includes the M1 solution space, i.e., $S(\mathrm{M} 1) \subseteq S(\mathrm{M} 2)$. Although theoretically expected, it should be noticed that in our previous work [8] with models with more degrees of freedom - referred to as M3 and M4 above the population-based approaches experienced several difficulties to efficiently explore broader upper-level spaces, yielding better results for the M3 model than for M4, in spite of M3 being a constrained M4. In the present work this has not happened: the algorithm proposed herein provides good results for M2, with values for $F$ systematically higher than the ones obtained with M1, also providing better figures (in any of the parameterizations) than the results obtained in [8] for M3 and M4, although $S(\mathrm{M} 2) \subseteq S(\mathrm{M} 3) \subseteq S(\mathrm{M} 4)$. 
- The improvement of the results from $30 \times 100$ to $30 \times 200$ is small, being slightly higher in M2 than in M1. The average improvement in M1 is $0.05 \%$, while in $\mathrm{M} 2$ is $0.08 \%$. The solution space $S(\mathrm{M} 2)$ is much larger than $S(\mathrm{M} 1)$ due to the combinatorial explosion of the price-periods combinations. This may explain why M2 benefits more than M1 from a longer search process; this may also justify the higher standard deviations in M2 than in M1. The improvement of $F$ from 100 to 200 generations does not seem relevant given the large increase in the computational effort, which doubles.

- The parameterization $40 \times 150$ does not produce better results, still looking slightly worse than $30 \times 200$, although 10 runs are not enough to support strong conclusions.

The non-parametric Kruskal-Wallis test was applied to assess whether the differences of the $F$ values obtained with the different parameterizations in each model are statistically significant, considering a significance level of $\alpha=0.01$. In both models, the differences are not statistically significant. The Mann-Whitney test comparing the results of M1 and M2 for the same parameterizations led to the conclusion that the differences are statistically significant in all the three cases.

The algorithms were run in a computer with an Intel Core i7-7700 CPU 3.6 GHz, 64 GB RAM. The computation time of each generation is similar for M1 and M2, which is on average less than 4" for the population size of 30 and 5" for the population size of 40 . The total computation time of one complete run is about 6'-6'30" for the parametrization $30 \times 100$ and about 12' $-13^{\prime} 30$ " for the parametrizations $30 \times 200$ and $40 \times 150$, which have similar computation times.

Given the results obtained in this experiment, we have adopted the $30 \times$ 100 parameterization because it presents a good compromise between solution quality and computation time. The best solution (maximum $F$ ) obtained for M1 has $F=6038.67$ and the best solution obtained for M2 has $F=6145.12$. We refer to these solutions as $\mathrm{Sol}_{\mathrm{M} 1}$ and $\mathrm{Sol}_{\mathrm{M} 2}$, respectively. The periods $\left[P_{i}^{1}, P_{i}^{2}\right]$, $i=1, \ldots, 6$, computed in $\mathrm{Sol}_{\mathrm{M} 2}$ are: [1-8], [9-12], [13-16], [17-48], [49-52], [53-96]. Fig. 3 compares the pre-defined periods in M1 with the ones obtained with M2, showing the times of the day $(\mathrm{h})$ that delimit the periods; $\{1, \ldots, 96\}$ corresponds to $00: 00 \mathrm{~h}-24: 00 \mathrm{~h}$ where $t=1$ represents the $t . u$. from $00: 00 \mathrm{~h}$ to $00: 15 \mathrm{~h}$, and so on. A significant difference between the periods of M1 and M2 can be observed: $P_{1}$ is the longer period in M1 ranging from 00:00h to 07:00h, while M2 defines three of the six periods from 00:00h to 04:00h. The longer period of M2 ranges from 13:00h to 24:00h.

Considering the periods of M2 in $\mathrm{Sol}_{\mathrm{M} 2}$ (shown in Fig. 3), we have further intensified the search in an attempt to improve the prices for these periods. Accordingly, these periods were fixed, and the M1 model was solved for these predefined periods. Five independent runs were performed from scratch (without injecting $\mathrm{Sol}_{\mathrm{M} 2}$ in the initial population) for the parameterization $N \times G=$ $30 \times 100$. The solutions obtained ranged from $F=6147.0$ to $F=6155.5$, with an average of $F=6151.1$, i.e., all improving $F\left(\mathrm{Sol}_{\mathrm{M} 2}\right)=6145.12$. The best solution $(F=6155.5)$ is also better that the one provided by running M2 for 


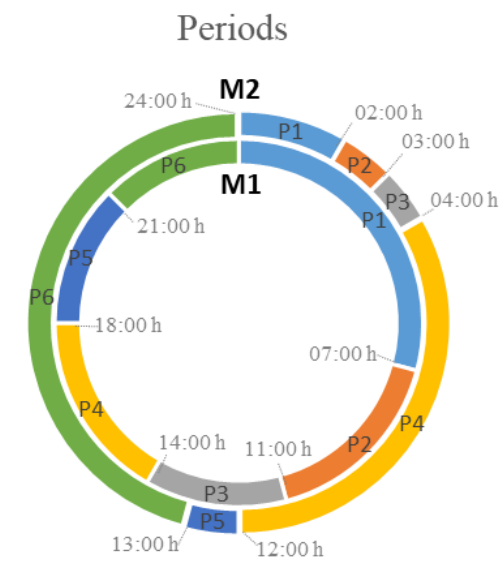

Fig. 3. Pre-defined periods in M1 and the best periods given by the algorithm for M2 in the $30 \times 100$ parameterization $\left(\mathrm{Sol}_{\mathrm{M} 2}\right)$

$30 \times 200$. This result suggests that it is better to execute the algorithm for the variable period model M2 during 100 iterations and then intensify the search for prices by using M1 for the best periods obtained than extending the search for periods and prices using M2 until 200 iterations. We have further experimented to run M2 for $N \times G=30 \times 500$. The best and average values obtained over 10 runs were $F=6152.85$ and $F=6126.82$, both being worse than the respective values obtained with the strategy M2+M1 (with $G=100$ in each one).

Let us denote by $\mathrm{Sol}_{\mathrm{M} 2+\mathrm{M} 1}$ the best solution obtained in the experiment $\mathrm{M} 2+\mathrm{M} 1$, which has the same periods as $\mathrm{Sol}_{\mathrm{M} 2}$ but slightly different prices. Table 2 shows the prices in $\mathrm{Sol}_{\mathrm{M} 1}$, $\mathrm{Sol}_{\mathrm{M} 2}$ and $\mathrm{Sol}_{\mathrm{M} 2+\mathrm{M} 1}$. Fig. 4 compares the best prices obtained for M1 (Sol ${ }_{\mathrm{M} 1}$ with a retailer's profit of 6038.67 ) and for M2 (Sol $\mathrm{M} 2+\mathrm{M} 1$ with a retailer's profit of 6155.50). Although a maximum of 6 different prices was allowed, solutions $\mathrm{Sol}_{\mathrm{M} 1}$ and $\mathrm{Sol}_{\mathrm{M} 2+\mathrm{M} 1}$ have fewer than 6 different prices (4 and 5 , respectively).

Table 2. Prices in the best solutions obtained for M1 and M2 in the $30 \times 100$ parameterization $\left(\mathrm{Sol}_{\mathrm{M} 1}\right.$ and $\mathrm{Sol}_{\mathrm{M} 2}$ ) and after the intensification of the search for M2 with $\mathrm{M} 1\left(\mathrm{Sol}_{\mathrm{M} 2+\mathrm{M} 1}\right)$

\begin{tabular}{|c|c|c|c|c|c|c|c|}
\hline & \multicolumn{6}{|c|}{ Prices $(€ / \mathrm{kWh})$} \\
\hline & & $P_{1}$ & $P_{2}$ & $P_{3}$ & $P_{4}$ & $P_{5}$ & $P_{6}$ \\
\hline \multirow{3}{*}{$\mathrm{Sol}_{\mathrm{M} 1}$} & Periods & [1-28] & {$[29-44]$} & {$[45-56]$} & {$[57-72]$} & {$[73-84]$} & [85-96] \\
\hline & Prices & 0.2976 & 0.2986 & 0.08 & 0.0803 & 0.0803 & 0.08 \\
\hline & $\overline{\text { Periods }}$ & {$[1-8]$} & {$[\overline{9}-\overline{1} 2]$} & {$[\overline{13}-\overline{1} \overline{6}]$} & {$[\overline{1} 7-4 \overline{8}]$} & {$[\overline{4} 9-5 \overline{2}]$} & {$[\overline{53}-\overline{96}]$} \\
\hline $\mathrm{Sol}_{\mathrm{M} 2}$ & Prices & 0.2689 & 0.2384 & 0.2701 & 0.2890 & 0.0804 & 0.0801 \\
\hline Sol $_{\mathrm{M} 2+\mathrm{M} 1}$ & Prices & 0.2683 & 0.2360 & 0.2793 & 0.2885 & 0.08 & 0.08 \\
\hline
\end{tabular}




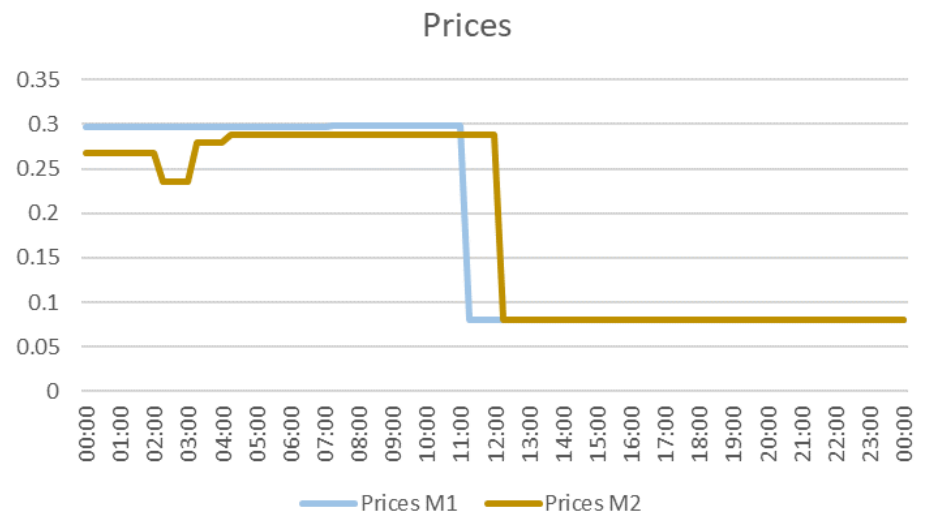

Fig. 4. Prices obtained for the models M1 (Sol 1 1 ) and $\mathrm{M} 2\left(\mathrm{Sol}_{\mathrm{M} 2+\mathrm{M} 1}\right)$.

\section{Conclusions}

This paper presented a comparison between two bilevel programming models to assist electricity retail companies to design optimal time-of-use tariffs. In the upper-level problem, the retailer maximizes the profit and in the lower-level problem the consumer minimizes the cost using his flexibility in the use of appliances in face of the time-differentiated prices. A bilevel model was previously presented by the authors in which the periods for setting the different prices were pre-defined and the aim was to determine the price values that maximize the retailer's profit. In this paper, a new more general model is proposed in which both the periods and prices are decision variables, thus leading to a very large search space for the upper-level problem due to the vast number of combinations periods-prices. To deal with this variable period model, a hybrid approach combining a genetic algorithm for the upper-level search with a mixed-integer linear programming solver to obtain optimal solutions to the lower-level problem has been developed. Specific encoding as well as crossover and mutation operators have been designed to make the most of the physical features of the problem.

The algorithm has been able to compute good quality solutions obtaining higher profit when the retailer can establish prices and periods over setting prices only, with a moderate computation effort. This information is of utmost importance for a retailer designing tariff options to offer consumers in very competitive electricity retail markets.

In order to cope with the complexity of the variable period model, additional experiments consisted of using the period configuration determined in this new model as an input of the pre-defined period model aiming to further improve prices. This intensification strategy proved useful since better solutions have been obtained in comparison with solutions found with a higher computation effort in the variable period model. 
Further work will involve a comprehensive study of time-of-use pricing problems vs. adequate features of algorithmic approaches, including using algorithms based on strategies other that the hybridization of metaheuristics with mathematical programming solvers.

Acknowledgments. This work was partially supported by projects UIDB/ 00308/2020 and by the European Regional Development Fund through the COMPETE 2020 Programme, FCT - Portuguese Foundation for Science and Technology and Regional Operational Program of the Center Region (CENTRO2020) within projects ESGRIDS (POCI-01-0145- FEDER-016434) and MAnAGER (POCI- 01-0145-FEDER-028040).

\section{References}

1. Dempe, S.: Foundations of bilevel programming. Springer Science \& Business Media. (2002)

2. Zugno, M., Morales, J.M., Pinson, P., Madsen, H.: A bilevel model for electricity retailers' participation in a demand response market environment. Energy Econ. 36, 182-197 (2013)

3. Meng, F.L., Zeng, X.J.: A bilevel optimization approach to demand response management for the smart grid. In: 2016 IEEE Congress on Evolutionary Computation (CEC). pp. 287-294 (2016).

4. Alves, M.J., Antunes, C.H., Carrasqueira, P.: A hybrid genetic algorithm for the interaction of electricity retailers with demand response. In: Applications of Evolutionary Computation, 19th European Conference (EvoApplications), LNCS, vol. 9597. pp. 459-474. Springer (2016).

5. Carrasqueira, P., Alves, M.J., Antunes, C.H.: Bi-level particle swarm optimization and evolutionary algorithm approaches for residential demand response with different user profiles. Inf. Sci. (Ny). 418-419, 405-420 (2017).

6. Soares, I., Alves, M.J., Antunes, C.H.: Designing time-of-use tariffs in electricity retail markets using a bi-level model - Computing bounds when the lower level problem cannot be exactly solved. Omega. In press (2019).

7. Aussel, D., Brotcorne, L., Lepaul, S., von Niederhäusern, L.: A Trilevel Model for Best Response in Energy Demand-Side Management. Eur. J. Oper. Res. 281, 299-315 (2020).

8. Soares, I.; Alves, M. J.; Antunes, C.H.: A bi-level optimization approach to define dynamic tariffs with variable prices and periods in the electricity retail market. In: Proceedings of the EUROGEN 2019, Springer ECCOMAS book series on Computational Methods in Applied Sciences. Accepted for publication (2020).

9. Marcotte, P., Savard, G.: A Bilevel Programming Approach to Optimal Price Setting. In: Zaccour, G. (ed.) Decision \& Control in Management Science: Essays in Honor of Alain Haurie. pp. 97-117. Springer US, Boston, MA (2002).

10. Labbé, M., Violin, A.: Bilevel programming and price setting problems. 4OR. 11, 1-30 (2013). 